\title{
Fasciola hepatica EN GANADO BOVINO DE CARNE EN SIQUIRRES Y LESIONES ANATOMO-HISTOPATOLÓGICAS DE HÍGADOS BOVINOS DECOMISADOS EN MATADEROS DE COSTA RICA
}

\author{
Carlos Ernesto Alpízar ${ }^{1 / *}$, Jaqueline Bianque de Oliveira**, Ana Eugenia Jiménez ${ }^{* *}$, \\ Jorge Hernández,* Alexis Berrocal ${ }^{* * *}$, Juan José Romero ${ }^{* * * *}$
}

Palabras clave: Parásitos, ganadería, trópico, patología, coprología, moluscos. Keywords: Parasites, cattle, tropics, pathology, coprology, mollusks.

Recibido: $29 / 01 / 13$

\section{RESUMEN}

El presente estudio fue realizado con el objetivo de determinar la infección por Fasciola hepatica ( $F$. hepatica) en una finca dedicada al ganado de carne en el cantón de Siquirres, provincia de Limón para caracterizar las lesiones más comunes de hígados parasitados, en 3 plantas de sacrificio de bovinos del país. De agosto del 2005 a agosto del 2006, fueron recolectadas muestras fecales de 577 bovinos de razas cebuinas con edades entre 6 meses y 2,5 años. Cada muestra fue sometida a la técnica coprológica de sedimentación; también se buscaron y recolectaron moluscos con el objetivo de identificar la(s) especie(s) involucrada(s) en la transmisión del parásito en la finca. Asimismo, se recolectaron muestras de hígados bovinos infectados con el parásito en 3 mataderos del Valle Central, para el análisis histopatológico. $F$. hepatica fue diagnosticada en 67 (11,3\%) de los 577 bovinos evaluados, y el porcentaje de infección fue mayor en agosto del $2005(31,6 \%)$ y agosto del 2006 $(26,2 \%)$. Además, los animales con edades entre 18 y 24 meses mostraron mayor frecuencia de infección (19,4\%), mientras que los animales de 12 a menos de 18 meses mostraron la menor

1 Autor para correspondencia. Correo electrónico: carlos.alpizar.solis@una.cr

* Departamento de Nutrición Animal, Escuela de Medicina Veterinaria, Universidad Nacional. Costa Rica.

Aceptado: 03/04/13

ABSTRACT
Fasciola hepatica infection in beef cattle
in Siquirres and anatomo-histopathologic
lesions of confiscated bovine liversin slaughters
in Costa Rica. This study was carried out in order
to determine Fasciola hepatica infection in a farm
dedicated to fattening beef cattle in Siquirres,
Limón province; also, to characterize the most
common lesions in parasitized bovine livers in
3 slaughterhouses in Costa Rica. Fecal samples
were collected from 577 zebu bovines aging
between 6 months and 2.5 years, from August
2005 to August 2006. Each sample was analyzed
by the fecal sedimentation technique. To identify
the species involved in parasite transmission, a
search and collection of mollusks in the farm was
also done. Besides, samples of infected bovine
livers were collected in 3 slaughterhouses of
the Central Valley, for histopathologic analysis.
F. hepatica was diagnosed in 67 (11.3\%) out
of 577 animals, with the highest percentage of
infection in August 2005 (31.6\%) and August
2006 (26.2\%). Animals aging between 18 and
24 months showed higher infection frequency
(19.4\%) than those between 12 and less than 18
months of age (2.4\%). Thiara tuberculata, the
** Cátedra de Parasitología, Escuela de Medicina
**terinaria, Universidad Nacional. Heredia, Costa Rica.
Laboratorio Histopatovet, Heredia, Costa Rica.
Programa de Investigación en Medicina Poblacional,
Nacional. Heredia, Costa Rica.


frecuencia de infección $(2,4 \%)$. Los caracoles identificados fueron de la especie Thiara tuberculata, que no fue identificada como vector de F. hepatica. Las principales lesiones en los hígados decomisados fueron atrofia moderada de lóbulos hepáticos, aumento de consistencia, engrosamiento de ductos hepáticos con presencia de calcificación, material mucoso y formas adultas del parásito. Histológicamente corresponden a colangiohepatitis necrótica con formación de depósitos cálcicos distróficos e hiperplasia de ductos biliares. Con base en los resultados obtenidos se pudo determinar la presencia de $F$. hepatica en los bovinos de la finca y de la zona, con marcado incremento de la infección en la época lluviosa, además del hallazgo colateral de Paramphistomum spp.

\section{INTRODUCCIÓN}

La fasciolosis es una enfermedad parasitaria de distribución mundial que afecta la salud de una amplia variedad de animales domésticos, silvestres e inclusive al hombre. Esto causa pérdidas económicas significativas para el sector pecuario mundial y tiene una gran importancia para la salud pública (Arroyo et ál. 1981). Es causada por el tremátodo digeneo Fasciola hepatica (F. hepatica), que sufre un desarrollo en hospedadores invertebrados y vertebrados como bovinos, equinos, suinos, roedores y humanos (Georgi y Georgi 1990).

El desarrollo óptimo de los huevos se da a temperaturas entre 10 y $30^{\circ} \mathrm{C}$ y al menos, con presencia de una pequeña cantidad de agua usualmente de movimiento lento (Carrada 2007) por lo que los climas tropicales húmedos y las condiciones de primavera y verano en zonas templadas son apropiadas para el desarrollo del parásito. El ciclo de vida requiere de ambientes húmedos, cálidos e inundados que favorezcan la presencia de moluscos de agua dulce principalmente del género Lymnaea (Carrada 2007). Comienza con la eliminación de huevos al medio ambiente en las heces snail specie found in the farm, was not identified as vector of $F$. hepatica. Main lesions in sampled infected livers were mild atrophy of hepatic lobes, tissue hardening, biliary ducts thickening with calcification, mucous material and adult parasites. Histologically, lesions were necrotic colangiohepatitis with dystrophic calcium deposits, together with biliary ducts hyperplasia. Based in these results, it was determined the presence of $F$. hepatica in bovines in this farm and in the region, with a marked infection increase in the rainy season, besides the collateral finding of Paramphistomum spp.

del hospedador vertebrado que posee formas adultas en los ductos biliares desde donde pasan al intestino delgado y luego a las heces (Bowman 2009). Si los excrementos son depositados en zonas inundadas o en cuerpos de agua, los huevos se desarrollan hasta miracidios que en el ambiente acuático, infectan a moluscos para transformarse en esporoquistes, redias y finalmente, cercarias que saldrán nuevamente a un medio acuático para enquistarse en plantas y dar origen a las metacercarias, fase larvaria infectante para vertebrados (Soulsby 1986, Cordero del Campillo y Rojo 2002). Malone (2002) indica que la mayoría de las infecciones ocurren por el pastoreo en zonas que tienen o están cerca de hábitats para moluscos que no son fijos y por lo menos permanecen húmedas durante la mitad del año.

En los vertebrados, el parásito adulto se alberga en el hígado e induce procesos inflamatorios crónicos (Thomson 1988, Bordin 1995) como fibrosis y calcificación de ductos hepáticos, aumento de la consistencia del parénquima, atrofia de lóbulo hepático ventral, formación de abscesos, y tractos hemorrágicos y necróticos (Malone 2002). Estos hallazgos post mortem 
pueden indicar las zonas geográficas con mayores problemas con el parásito cuando el ganado proveniente de ciertos lugares es llevado a matanza y presenta frecuencias altas del parásito (Kithuka et ál. 2002).

Los hospedadores intermediarios son moluscos de la familia Lymnaeidae (Georgi y Georgi 1990) y en Costa Rica se han identificado Pseudosuccinea columella y Fossaria cubensis como posibles involucradas en la transmisión en Turrialba (Ramón et ál. 1968, Arroyo et ál. 1981), cantón en donde se han realizado la mayoría de estudios de la enfermedad en el país.

Rapsch et ál. (2006) estimaron, mediante el uso de inspección visual post mortem, sedimentación fecal, ELISA y detección microscópica post mortem de huevos en bilis, una prevalencia real de un $18 \%$ en Suiza, mientras que Salimi et ál. (2005) en el Reino Unido determinaron mediante un ELISA en la leche, que la prevalencia era de alrededor de $48 \%$ en Inglaterra y de $86 \%$ en Gales. En Kenya, Kithuka et ál. (2002) lograron determinar durante 10 años, prevalencias de entre $4 \%$ y $16 \%$ por medio de examen post mortem del ganado.

Debido a las condiciones favorables para el desarrollo del parásito en el caribe costarricense, a los frecuentes reportes de animales infectados detectados en el matadero que provienen de esa zona y a la falta de estudios al respecto en otras zonas de la región atlántica de Costa Rica, se realizó este trabajo con el objetivo de determinar la infección por $F$. hepatica en una finca de ganado bovino de carne del cantón de Siquirres, así como describir las lesiones anatomo-histopatológicas, y su frecuencia en hígados de bovinos decomisados en mataderos de Costa Rica.

\section{MATERIALES Y MÉTODOS}

\section{Área y momento del estudio}

El estudio se llevó a cabo entre agosto de 2005 y agosto de 2006, en la localidad de Caño Blanco de Siquirres, provincia de Limón $\left(10^{\circ} 08^{\prime} 45^{\prime \prime}\right.$ latitud norte y $83^{\circ} 29 ' 50^{\prime \prime}$ longitud oeste), en una finca con una extensión de 800 hectáreas, con una topografía prácticamente plana, irrigada por un riachuelo y dedicada al desarrollo y engorde de ganado. La finca posee, en el momento del estudio, aproximadamente 800 animales de las razas Brahman y Nelore.

\section{Análisis coprológicos}

Muestras fecales individuales de aproximadamente $200 \mathrm{~g}$ fueron recolectadas directamente del recto de 577 animales con edades entre los 6 meses y 2,5 años. Dichos muestreos fueron realizados cada 2 meses durante un año que comprendió de agosto de 2005 a agosto de 2006, para un total de 7 muestreos. Las muestras fueron conservadas en bolsas plásticas de $50 \mathrm{~cm}$ por $20 \mathrm{~cm}$, a una temperatura de $4^{\circ} \mathrm{C}$ y trasladadas al Laboratorio de Parasitología de la Escuela de Medicina Veterinaria (EMV) de la Universidad Nacional en donde se realizó la técnica de sedimentación fecal según Hernández (2004) para buscar huevos del parásito. Esta consiste en tomar aproximadamente 2 a $4 \mathrm{~g}$ de heces y macerarlos en agua para luego tamizar hasta llenar una copa de sedimentación y dejarla en reposo durante 15 minutos, posteriormente a esto se decanta es sobrenadante y se vuelve a llenar la copa con agua para dejar en reposo 15 minutos más; finalmente se decanta el sobrenadante y se agregan 2 gotas de azul de metileno al sedimento para crear un contraste al microscopio que facilita la observación de los huevos del parásito. El sedimento se observa al microscopio a un aumento de 10X para buscar e identificar los huevos de los parásitos.

\section{Identificación de caracoles}

La búsqueda de los moluscos se realizó mediante el tamizado del suelo y el lodo de charcas y márgenes de riachuelos cada vez que se visitó la finca. Para esa labor se utilizó un pazcón grande y se revisó el sedimento luego de lavarlo, para verificar la presencia de los moluscos. Los moluscos hallados fueron trasladados en tubos cónicos plásticos de $50 \mathrm{ml}$ al laboratorio de 
Hidrología Ambiental de la Escuela de Biología de la Universidad Nacional para su identificación visual. Adicionalmente, en el Laboratorio de Parasitología (EMV) se determinó la existencia de estadíos larvarios del parásito en los especímenes, manteniéndolos 1 a 2 días en cajas de Petri con agua destilada, para observar las cajas directamente bajo el estereoscopio.

\section{Análisis macro y microscópico de hígados parasitados}

En total, se recolectaron 16 hígados de bovinos infectados en 3 plantas de sacrificio del Valle Central procedentes de todo el país, que fueron trasladados en contenedores a una temperatura de $4^{\circ} \mathrm{C}$ hasta el Servicio de Patología de la EMV, en donde se realizó una identificación macroscópica de las lesiones. Sin embargo, estos órganos no pertenecen a ninguno de los animales de la finca en estudio. Los especímenes con lesiones macroscópicas más significativas (3 órganos), se sometieron a estudio microscópico luego de utilizar la tinción básica de hematoxilina y eosina. Además, se describieron las lesiones al corte de los mismos órganos sometidos a estudio microscópico para evidenciar lesiones no visibles en la superficie de los hígados.

\section{Análisis estadísticos}

Se calcularon los porcentajes de muestras positivas a $F$. hepatica, según la edad de los animales, para el acumulado del periodo. Tanto para Paramphistomum spp., como para F. hepatica. Se calcularon los porcentajes de muestras positivas por mes de muestreo. Complementariamente, se realizaron pruebas de diferencias de proporciones de positividad, entre e intra especies, por mes de muestreo. Finalmente, para cada parásito, se comparó el porcentaje de muestras positivas entre meses lluviosos (junio, agosto, octubre) y meses secos (febrero, abril, diciembre). Los cálculos se realizaron con un valor de significancia $(\alpha) 0,05$, por medio del programa Infostat versión 1.1. (Rienzo et ál. 2002).

\section{RESULTADOS}

\section{Análisis coprológico}

En total, se encontraron 65 animales infectados de 577 evaluados (11,3\%) por F. hepatica. Según edades, se observó diferencia significativa entre los porcentajes de infección para los animales con rango de edad de 12 a 18 meses, que fue el más bajo respecto a animales con edades fuera de ese rango. Animales entre 18 a 24 meses de edad presentaron mayor frecuencia de infección (Cuadro 1).

Los porcentajes más altos de infección por $F$. hepatica se observaron en agosto del 2005 (31,6\%) y de 2006 (26,2\%), y los porcentajes más bajos en diciembre de $2005(3,8 \%)$ y abril de $2006(3,0 \%)$ (Cuadro 2). Los porcentajes

Cuadro 1. Comportamiento por edades de la infección por Fasciola hepatica en la finca evaluada.

\begin{tabular}{cccc}
\hline Edad (meses) & No $^{\text {de muestras }}$ & $\mathrm{N}^{\mathrm{o}}$ de positivos & Porcentaje \\
\hline$<12$ & 76 & 8 & $10,5^{\mathrm{a}}$ \\
$12-<18$ & 204 & 5 & $2,5^{\mathrm{b}}$ \\
$18-24$ & 237 & 46 & $19,4^{\mathrm{a}}$ \\
$>24$ & 60 & 6 & $10,0^{\mathrm{a}}$ \\
Total & 577 & 65 & 11,3 \\
\hline
\end{tabular}

Los literales distintos indican diferencia estadística entre los rangos de edades, con un valor de $\alpha=0,05$. Los rangos con la misma letra no presentan diferencia estadísticamente significativa entre sí. 


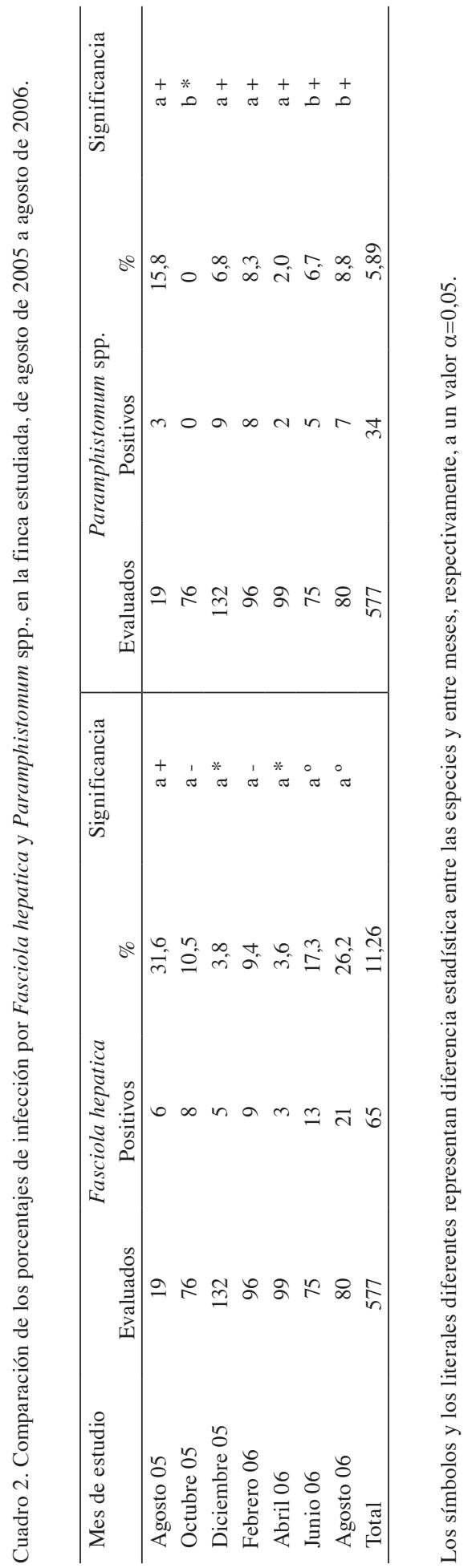

Agronomía Costarricense 37(2): 7-16. ISSN:0377-9424 / 2013 
de infección (acumulados) de los meses de la estación lluviosa son mayores ( $\mathrm{p}<0,001)$, al ser 19,2\% (48/250), en comparación con los meses de la estación seca que fue de 5,2\% (17/327). Sin embargo entre meses de una misma estación la diferencia es mucho menor.

Se logró determinar también la presencia de huevos del tremátodo Paramphistomum spp., en un total de $5,9 \%$ de los animales $(34 / 577)$, con los mayores porcentajes agosto de 2005 y 2006 (15,8\% y 8,8\% respectivamente), y los menores porcentajes en octubre de 2005 y abril de 2006 ( $0,0 \%$ y $2,0 \%$ respectivamente). En el caso de este parásito no hubo diferencias significativas entre los acumulados de los meses lluviosos respecto a los de la época seca (Cuadro 2). Se notó también diferencia significativa entre los porcentajes de infección de $F$. hepatica y Paramphistomun spp., en octubre de 2005, junio y agosto de 2006. El porcentaje global de infección por Paramphistomum spp., representó la mitad del porcentaje de infección mostrado por $F$. hepatica. No obstante, estos resultados muestran un comportamiento similar entre sí, con una tendencia al aumento de la frecuencia de infección en agosto.

Todos los animales infectados con Paramphistomun spp., fueron mayores a 1 año y el grupo etario con mayor porcentaje de animales infectados fue el de más de 1,5 años hasta 2 años con $52 \%$ de los animales infectados, seguido de los animales de 1 año hasta 1,5 años con 37\%; de último, los animales mayores a 2 años con un $11 \%$ de los infectados. Estos porcentajes corresponden al 2,4\%, $1,7 \%$ y $0,5 \%$ de todos los animales evaluados, respectivamente.

\section{Identificación de caracoles}

Los caracoles encontrados pertenecen a la especie Thiara tuberculata (Figura 1), en los que se identificaron cercarias del tipo Parapleurolophocercaria, que no corresponden con el tipo de F. hepatica.

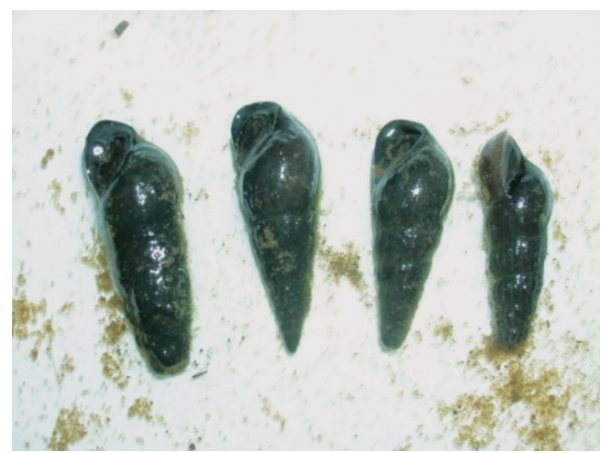

Fig. 1. Ejemplares del molusco Thiara tuberculata encontrados en la finca estudiada.

\section{Caracterización anatomo-histopatológica de las lesiones en hígados decomisados}

Macroscópicamente, las lesiones encontradas en hígados decomisados de bovinos tanto en cara parietal como en cara visceral fueron ductos biliares prominentes (11/16), atrofia del lóbulo izquierdo (8/16), fibrosis de los ductos biliares y del parénquima (6/16), hiperplasia de los linfonodos (5/16), calcificaciones en el lumen de ductos biliares (3/16), presencia de formas adultas del parásito $(2 / 16)$ y abscesos hepáticos (1/16).

Al corte de los órganos se observó dilatación de los ductos biliares (2/3), presencia de material mineralizado en los ductos biliares $(1 / 3)$, presencia de formas adultas del parásito (2/3) y fibrosis del tejido hepático (1/3) (Figura 2).

Microscópicamente las lesiones fueron hiperplasia de linfonodos (1/3), fibrosis de los ductos biliares (3/3), presencia periductular de macrófagos (1/3) y eosinófilos (1/3), fibrosis del tejido hepático (1/3), hipervascularización del parénquima $(1 / 3)$ y calcificación de los ductos biliares $(1 / 3)$. 


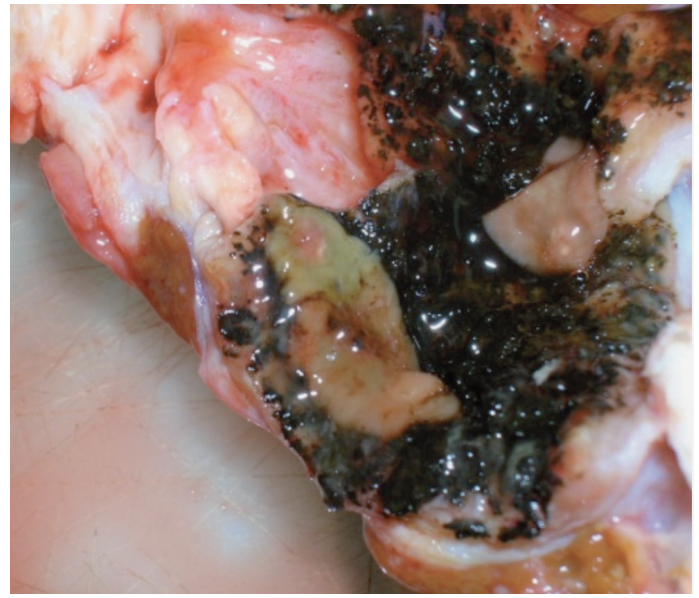

Fig. 2. Ducto biliar de hígado decomisado parasitado por Fasciola hepatica, con presencia de formas adultas.

\section{DISCUSIÓN}

Este es el primer reporte de la presencia de Fasciola hepatica y Paramphistomum spp., en el cantón de Siquirres, y corrobora lo que refieren los ganaderos de la zona quienes indican que han sufrido notables pérdidas económicas debido, principalmente, al decomiso de los hígados de los animales durante la inspección de vísceras en el matadero. Paralelamente a este estudio, se estimaron las pérdidas económicas que significa, en términos generales el decomiso de los hígados en las plantas de sacrificio según el precio en el que se venden los órganos en el mercado (Alpízar 2008). Sin embargo, la enfermedad se puede traducir también en importantes pérdidas económicas por disminución de la producción, problemas reproductivos e inclusive muerte de animales (Mora et ál. 1980, Becerra 2001, Schweizer et ál. 2005).

Los porcentajes globales de infección obtenidos en este estudio, mediante la prueba de sedimentación fecal, son menores a los reportados por Herrera (1979) y Cartín y Chang (1983) en las localidades de Agua Caliente de Cartago y Santa Cruz de Turrialba, los cuales fueron de 17\% y $42,2 \%$ respectivamente.
Estos autores utilizaron también técnicas coproparasitológicas y son los principales reportes del parásito previos realizados en ganado en el país. Pero, otros autores reportan porcentajes similares e inclusive mayores en Venezuela (Nieves et ál. 2005) y Brasil (Marques et ál. 2003), con $39 \%$ y $63,3 \%$ respectivamente. Mungía et ál. (2007) encontraron porcentajes de infección en bovinos muy similares a los encontrados en la presente investigación $(10,2 \% \pm 1,1)$ al utilizar también, la técnica de sedimentación en un trabajo realizado en zona semidesértica del Estado de Sonora en México en el que igualmente determinaron que los animales con menos de 3 años tenían mayores porcentajes de infección. Al respecto, dado el ciclo de vida del parásito, son muchas las variables que pueden producir diferencias en los porcentajes de infección con $F$. hepática como las mencionadas en estos estudios, tales como la técnica diagnóstica usada para la detección, condiciones climáticas, época del año, especie del hospedador, dosis infectante, presencia de parásitos en etapa prepatente el tamaño de la muestra y la cantidad de heces recolectadas para los análisis (Mungía et ál. 2007), entre otras.

Los meses en los que se detectó mayor porcentaje de infección fueron agosto de 2005 y agosto de 2006 y la época del año con mayor porcentaje de infección es la época lluviosa según los datos obtenidos para cada mes en estudio, pues de agosto a diciembre de 2005, de 227 muestras fecales analizadas 19 resultaron positivas $(8,3 \%)$, y de junio a agosto de 2006 el 22\% de las 155 muestras fueron positivas, a diferencia de la época seca (febrero y abril de 2006) en la que se analizaron 195 muestras y sólo 12 (6\%) fueron positivas. Esto podría indicar que los animales adquieren el parásito al menos 3 a 4 meses antes de los meses con mayor porcentaje de infección, ya que Soulsby (1986), Cordero del Campillo y Rojo (2002) y Suon et ál. (2006) han indicado que los adultos de este tipo de parásitos pueden empezar la postura 3 a 4 meses posteriores a la infección del animal, en condiciones óptimas para su desarrollo. Además, este periodo puede coincidir con la presencia de condiciones favorables para 
la infección según datos de Cruz et ál. (2005), quienes indican que en México de mayo a julio se presentan temperaturas entre $22^{\circ} \mathrm{C}$ y $26^{\circ} \mathrm{C}$, una humedad relativa promedio de $45 \%$ y que dichas condiciones son aptas para la infección del ganado con el tremátodo. En nuestro país, según el Instituto Meteorológico Nacional (2007), abril en la Región Atlántica, históricamente desde 1970, ha reportado temperaturas entre 22,0 y $30,1^{\circ} \mathrm{C}$ y una baja cantidad de días con lluvias, al igual que muy bajas precipitaciones. Esta información puede sugerir que condiciones más secas, luego de periodos de lluvias, favorecen el pastoreo de los animales y a la vez la infección del ganado con las metacercarias que se ven favorecidas por un ambiente húmedo para alcanzar las pasturas $\mathrm{y}$ lograr su enquistamiento.

Es notable también, que los animales entre 18 y 24 meses presenten significativamente un mayor porcentaje de infección; hecho que coincide con los datos reportados por Cartín y Chang (1983) en Turrialba. Además Kleiman et ál. (2007), en Argentina, reportaron un $81 \%$ de infección en novillas. Si la infección se da hacia los 18 meses, esto en parte se puede explicar basado en los hallazgos de Wiedosari et ál. (2006) que señalan que es posible que, alrededor de las 24 semanas posteriores a la infección, algunas razas de ganado desarrollen cierta resistencia al parásito y, consecuentemente, sean capaces de eliminar el adulto. No obstante, no existe evidencia en este estudio, ni en otros, que la infección de los animales se da hacia los 18 meses; lo que si se observó, es que los animales con edad entre 12 y 18 meses presentaron el menor porcentaje de infección.

La especie de moluscos identificada en este estudio (Thiara tuberculata) no ha sido reportada como hospedador intermediario o como posible hospedador intermediario de F. hepatica en ningún estudio y aunque pudo verificarse la presencia de cercarias en los mismos, éstas no eran del tipo gimnocéfala producido $F$. hepática, por tanto, no puede considerarse como el vector del tremátodo en la finca. Sin embargo, en Costa Rica, Tonn et ál. (1964) encontraron cercarias gimnocéfalas en moluscos de los géneros Heliosoma, Stenophysa y Physa y lograron definir a éstos géneros como vectores del parásito, al menos en el distrito de La Unión de Cartago. Posteriormente, se determinó también que las especies Pseudosuccinea columella y Fossaria cubensis son vectores de $F$. hepatica en la provincia de Cartago (Ramón et ál. 1968, Cartín y Chang 1983).

La presencia de Paramphistomun en los animales muestreados es un hecho que debe investigarse más a fondo, lo que no fue parte de los objetivos de este estudio, pero deja claro que las condiciones de la región favorecen el ciclo de vida de los tremátodos, que hay moluscos presentes y posiblemente sean hospedadores compartidos entre las especies de parásitos, además que la enfermedad está presente aunque no existen reportes previos.

Por otra parte, la mayor cantidad de lesiones encontradas en los hígados bovinos estudiados son de tipo inflamatorias y crónicas. Dichas lesiones consistieron especialmente en fibrosis periductular, calcificación e hiperplasia de los ductos biliares (Figura 2), no obstante no muestran una ubicación determinada en los órganos como lo indican otros autores (Thomson 1988, Bordin 1995). Aunque en este trabajo no se estudió una relación entre la presencia de formas adultas de F. hepatica y el tipo y ubicación de lesiones en los órganos sometidos a análisis macro y microscópico, el estudio de Marcos et ál. (2007) logró establecer alguna relación entre la severidad de las lesiones y el número de formas adultas en los hígados. La presencia de macrófagos y eosinófilos no fue frecuente, posiblemente debido a que los órganos estudiados presentaron lesiones de tipo crónico, que resultan de la resolución mediante procesos fibróticos de las lesiones y fases agudas, causadas por las migraciones de los parásitos a través del parénquima y de las obstrucciones de los ductos biliares que son las fases que mayor infiltración celular inflamatoria provocan (McGavin et ál. 2009). Además, se ha reportado que $F$. hepatica puede disminuir la respuesta inmune celular contra algunas especies de bacterias indirectamente a través de la inhibición de células Th1 por las células Th2 específicas 
para $F$. hepatica, y directamente de forma independiente de los mecanismos de inmunidad celular a través de la actividad de proteinasas sobre las moléculas inmunológicas como interleucinas (Dorchies 2006), lo que justifica en parte la pobre respuesta inmunológica celular contra las formas migratorias del parásito en los hígados.

\section{CONCLUSIONES}

La infección con $F$. hepatica en bovinos en nuestro país puede tener una distribución más amplia de la que se pensaba hace algunos años, según los reportes previos a este estudio. Además, está presente en nuestro país Paramphistomum spp. Según lo anterior, es necesario el desarrollo y empleo de estrategias de identificación y control de la enfermedad en animales y en regiones del país así como de un estudio más exhaustivo para conocer la dinámica de la infección tanto de $F$. hepatica como de Paramphistomum spp., que incluya las épocas de infección y las especies de caracoles involucradas. Las lesiones típicas que se encuentran en el hígado de animales infectados en nuestro país son lesiones inflamatorias de tipo crónico. Estudios posteriores también deben contemplar las pérdidas económicas causadas por el decomiso de órganos en mataderos, así como los parámetros para evaluar su efecto sobre la reproducción.

El control de la infección por $F$. hepatica comprende un sistema integral que incluye la disminución de adultos y la eliminación de huevos al ambiente mediante principios farmacológicos, que a la vez disminuye la cantidad de fases larvarias en los vectores (Gray y Parr 2000), esto disminuye la probabilidad de infección en el ganado, así como la evaluación de la eficiencia de éstos antihelmínticos (Elitok et ál. 2006) debido al desarrollo de resistencia del trematodo, sin embargo, es más eficiente el control del hábitat de los moluscos transmisores, especialmente en zonas con altas precipitaciones, además de suelos y geografía propensos a la formación de charcos e inundaciones que favorecen el desarrollo de los caracoles y con presencia comprobada de los tremátodos como en el caso de la finca estudiada.

\section{LITERATURA CITADA}

ALPÍZAR C. 2008. Infección por Fasciola hepatica en bovinos de carne de una finca en Siquirres e impacto económico del decomiso de hígados en tres mataderos del área metropolitana de Costa Rica. Tesis de licenciatura, Universidad Nacional, Heredia, Costa Rica. 40 p.

ARROYO R., MORA J., MOLINA S., TROPER L., AMADOR A. 1981. Fascioliasis hepática humana en Costa Rica. Revista Costarricense de Ciencias Médicas 2(1):35-57.

BECERRA M. 2001. Consideraciones sobre estrategias sostenibles para el control de Fasciola hepatica en Latinoamérica. Revista Colombiana de Ciencias Pecuarias 14(1):228-235.

BORDIN E. 1995. Revisão da anatomia patológica de fasciolose bovina. A hora veterinária 15(1):33-35.

BOWMAN D. 2009. Georgi's parasitology for veterinarians. $6^{\text {th }}$ ed. Saunders, Estados Unidos. $451 \mathrm{p}$.

CARRADA T. 2007. Fasciola hepatica: Ciclo biológico y Potencial biótico. Revista Mexicana de Patología Clínica 54(1):21-27.

CARTÍN M., CHANG E. 1983. Diagnóstico y control de la fasciolasis bovina en el distrito de Santa Cruz de Turrialba, provincia de Cartago. Tesis de licenciatura, Universidad Nacional, Costa Rica. 49 p.

CORDERO M., ROJO F. 2002. Parasitología Veterinaria. Mc Graw-Hill, España. 968 p.

CRUZ I., IBARRA F., QUINTERO M., NARANJO E., LECUMBERRI J., CORREA D. 2005. Seasonal transmission of Fasciola hepatica in cattle and Lymnaea (Fossaria) humilis snails in central Mexico. Parasitology Research 95(4):283-286.

CULLEN M., MACLACHLAN J. 2009. Liver, biliary system, and exocrine pancreas, pp. 81-124. In: M. McGavin, W. Carlton and J. Zachary (eds.). Thomson's Special Veterinary Pathology. Mosby, United States.

DORCHIES P. 2006. Flukes: old parasites but new emergence. Proceedings of the XXIV World Buiatrics Congress. Nice, France. Consultada 16 de Julio de 2013 en http://www.ivis.org/proceedings/wbc/wbc2006/ dorchies.pdf?LA=1

ELITOK B., ELITOK Ö., KABU M. 2006. Field trial in comparative efficacy of four fasciolicides against natural liver fluke infection in cattle. Vet. Parasitol. 135:279-285.

GEORGI J., GEORGI M. 1990. Parasitology for veterinarians. W.B Saunders Company, New York, United States. $412 \mathrm{p}$.

HERNÁNDEZ J. 2004. Técnicas parasitológicas. Cátedra de Parasitología y Enfermedades Parasitarias, Universidad Nacional, Costa Rica. 15 p.

HERRERA R. 1979. Diagnóstico de fasciolosis: estudio comparativo entre la intradermorreacción, examen coproparasitológico y contrainmunoelectroforesis. 
Tesis de licenciatura. Universidad Nacional, Costa Rica. $50 \mathrm{p}$.

INSTITUTO METEOROLÓGICO NACIONAL (IMN). 2007. Promedio de las precipitaciones y temperaturas ambientales de Limón en los últimos 42 años. Instituto Meteorológico Nacional, San José, Costa Rica. 1 de enero de 2012, www.imn.ac.cr

KITHUKA J., MAINGI N., NJERUH F., OMBUI J. 2002. The prevalence and economic importance of bovine fasciolosis in Kenya: an analysis of abattoir data. Onderstepoort Journal of Veterinary Research 69(4):255-262.

KLEIMAN F., PIETROKOVSKY S., PREPELITCHI L., CARBAJO A., WISNIVESKY C. 2007. Dynamics of Fasciola hepatica transmission in the andean patagonian valleys, Argentina. Veterinary Parasitology 145(3-4):274-86.

MALONE J. 2002. Diseases of the Hepatobiliary System: liver flukes in ruminants, pp. 805-808. In: B.P. Smith (ed.). Large Animal Internal Medicine. Mosby.

MARCOS L., YI P., MACHICADO A., ANDRADE R., SALMAVIDES F., SÁNCHEZ J., TERASHIMA A. 2007b. Hepatic fibrosis and Fasciola hepatica infection in cattle. Journal Helminthology 81(4):381386.

MARQUES T., MARCIA S., SCHROFERNEKER M. 2003. Fasciola hepatica infection in cattle and buffaloes in the State of Rio Grande do Sul, Brazil. Parasitología Latinoamericana 58(3-4):169-172.

MORA J., ARROYO R., MOLINA S., TROPER L., IRÍAS E. 1980. Nuevos aportes sobre el valor de la fasciolina: estudio en un área endémica de Costa Rica. Boletín de la Oficina Sanitaria Panameña 89(5):409-414.

MUNGÍA J., IBARRA F., DUCOING A., MONTENEGRO N., QUIROZ H. 2007. Prevalence of Fasciola hepatica (ELISA and fecal analysis) in ruminants from a semi-desert area in the northwest of Mexico. Parasitology Research 101:127-130.

NIEVES E., RONDON M., ZAMORA E., SALAZAR M. 2005. Fasciola hepatica (Trematoda: Fasciolidae) en la zona alta de Mérida, Venezuela. Revista Electrónica REDVET 6:1-12.

PARR S., GRAY J. 2000. A strategic dosing scheme for the control of fasciolosis in cattle and sheep in Ireland. Vet. Parasitol. 88:187-197.
RAMÓN R., ARROYO G., MUÑOZ G., DELGADO E. 1968. Estudio preliminar sobre Fasciola hepatica en Costa Rica. Revista de Biología Tropical 15(1):137142.

RAPSCH C., SCHWEIZER G., GRIMM F., KOHLER L., BAUER C., DEPLAZES P., BRAUN U., TORGERSON P. 2006. Estimating the true prevalence of Fasciola hepatica in cattle slaughtered in Switzerland in the absence of an absolute diagnostic test. International Journal for Parasitology 36(10-11):1153-1158

RIENZO J.A., BALZARINI M.G., ROBLEDO C., GONZALEZ L., TABLADA M., GUZMAN W., CASANOVES F. 2002. Infostat/P 1.1. Facultad de Ciencias Agropecuarias; Estadística y diseño. Córdoba, Argentina.

SALIMI R., DANIEL R., FESLTEAD S., CRIPPS P., MAHMOODY H., WILLIAMS D. 2005. Prevalence of Fasciola hepatica in dairy herds in England and Wales measured with an ELISA applied to bulk-tank milk. Veterinary Record 156:729-731.

SCHWEIZER G., BRAUN U., DEPLAZES P., TORGERSON P. 2005. Estimating the financial losses due to bovine fasciolosis in Switzerland. The Veterinary Record 157:188-193.

SOULSBY E. 1986. Parasitología y enfermedades parasitarias en los animales domésticos. Interamericana, México D.F. 823 p.

SUON S., HOL D., SIEK S., McLEAN M., COPEMAN B. 2006. Seasonal differences in the incidence of infection with Fasciola gigantica in cambodian cattle. Tropical Animal Health and Production 38(1):23-8.

THOMSON R. 1988. Special Veterinary Pathology. Mosby, Pennsylvania, E.U.A. 661 p.

TONN R., HUNTER G., ALFARO M., ZÚÑIGA J., REDMOND D. 1964. Seasonal incidence of larval trematodes in Costa Rica. Revista de Biología Tropical 12:59-65.

WIEDOSARI E., HAYAKAWA H., COPEMAN B. 2006. Host differences in response to trickle infection with Fasciola gigantica in buffalo, ongole and bali calves. Tropical Animal Health and Production 38(1):43-53.

Todos los derechos reservados. Universidad de Costa Rica. Este artículo se encuentra licenciado con Creative Commons Reconocimiento-NoComercial-SinObraDerivada 3.0 Costa Rica. Para mayor información escribir a rac.cia@ucr.ac.cr 